\title{
A New Ceramide (Rumexamide) and Other Chemical Constituents from Rumex abyssinicus Jacq (Polygonaceae): Isolation, Characterization, Antibacterial Activities and Chemophenetic Significance
}

\section{Léonel Donald Feugap Tsamo1,2, Lorette Victorine Yimgang3, Steven Collins N. Wouamba4, Pierre Mkounga ${ }^{2}$, Augustin Ephrem Nkengfack ${ }^{2}$, Laurence Voutquenne-Nazabadioko ${ }^{5}$, David Ngnokam ${ }^{1}$, Bruno Ndjakou Lenta ${ }^{4}$, Norbert Sewald ${ }^{6}$}

${ }^{1}$ Research Unit of Applied and Environmental Chemistry, Department of Chemistry, Faculty of Science, University of Dschang, Dschang, Cameroon

${ }^{2}$ Department of Organic Chemistry, Faculty of Science, University of Yaoundé I, Yaoundé, Cameroon

${ }^{3}$ Antimicrobial Agents Unit, Laboratory for Phytobiochemistry and Medicinal Plants Studies, University of Yaoundé I, Yaoundé,

Cameroon

${ }^{4}$ Department of Chemistry, Higher Teacher Training College, University of Yaounde I, Yaoundé, Cameroon

${ }^{5}$ Groupe Isolement et Structure, Institut de Chimie Moléculaire de Reims (ICMR), Reims, France

${ }^{6}$ Organic and Bioorganic Chemistry, Faculty of Chemistry, Bielefeld University, Bielefeld, Germany

Email: *dngnokam@yahoo.fr

How to cite this paper: Tsamo, L.D.F., Yimgang, L.V., Wouamba, S.C.N., Mkounga, P., Nkengfack, A.E., Voutquenne-Nazabadioko, L., Ngnokam, D., Lenta, B.N. and Sewald, N. (2021) A New Ceramide (Rumexamide) and Other Chemical Constituents from Rumex abyssinicus Jacq (Polygonaceae): Isolation, Characterization, Antibacterial Activities and Chemophenetic Significance. Advances in Biological Chemistry, 11, 266-282.

https://doi.org/10.4236/abc.2021.115018

Received: September 16, 2021 Accepted: October 26, 2021 Published: October 29, 2021

\begin{abstract}
The chemical study of Rumex abyssinicus Jacp (Polygonaceae) led to the isolation of a new ceramide named $(R)-2$ '-hydroxy- $N$-[(2S,3S,4R,16E)-1,3,4-trihydroxyhexacos-16-en-2-yl]hexadecanamide (rumexamide) (1) together with sixteen known compounds: bis-(2-ethylhexyl) phthalate (2), chrysophanol (3), physcion (4), citreorosein (5), emodin (6), chrysophanein (7), physcionin (8), lupeol (9), 3 $\beta, 28$-dihydroxylup-20(29)-ene (10), 3 $\beta$-dihydroxylup-20(29)-en-28oic acid (11), oleanolic acid (12), ergosta-6,22-diene-3,5,8-triol (13), stigmastane-3,6-dione (14), a mixture of $\beta$-sitosterol (15) and stigmasterol (16), and stigmasterol 3-O- $\beta$-D-glucoside (17). Their structures were determined by interpretation of their spectroscopic $1 \mathrm{D}$ NMR $\left({ }^{1} \mathrm{H}\right.$ and ${ }^{13} \mathrm{C}$ NMR), 2D NMR (COSY ${ }^{1} \mathrm{H}-{ }^{1} \mathrm{H}, \mathrm{HSQC}$ and $\mathrm{HMBC}$ ) data in conjunction with mass spectrometry (TOFESIMS and HR-TOFESIMS) and by comparison with those reported in the literature. Among all the known compounds, twelve (2, 5, 8-17) were firstly isolated from Rumex abyssinicus, seven (2, 10-14 and 17) from the genus Rumex and three $(13,14,17)$ from family Polygonaceae. The in vitro anti-
\end{abstract}


Copyright $\odot 2021$ by author(s) and Scientific Research Publishing Inc. This work is licensed under the Creative Commons Attribution International License (CC BY 4.0).

http://creativecommons.org/licenses/by/4.0/ bacterial activities of extracts $(\mathrm{MeOH}, n-\mathrm{BuOH}$ and EtOAc), as well as compounds 9, 11, 12, $15+16$ and 17 against pathogenic bacteria (Staphylococcus aureus ATCC 43300, Shigella flexneri NR 518, Klebsiella pneumoniae ATCC 700603, Escherichia coli ATCC 25922), were performed using the broth microdilution method and the results show that, extracts were not active (MIC > $1000 \mu \mathrm{g} / \mathrm{mL}$ ) while compounds were weakly or not active (MIC $\geq 500 \mu \mathrm{g} / \mathrm{mL}$ ) against all bacteria strains. Furthermore, the chemophenetic relationships of the isolated compounds and their significances were discussed.

\section{Keywords}

Polygonaceae, Rumex abyssinicus, Rumexamide, Antibacterial Activities, Chemophenetic Significance

\section{Introduction}

Since their discoveries, antibiotics have been essential in the treatment of diseases caused by pathogens (especially bacterial infections) which have helped in extending the average life expectancy. However, their overexploitation has caused the appearance and increase in the rate of resistance of microorganisms to said antibiotics [1]. It is well established that this resistance is nowadays responsible for nearly 7 million deaths per year and it is estimated that it will reach to 10 million deaths per year by 2050 [2]. Thus, in order to overcome this state of affairs, efforts are being made to find new antimicrobial agents such as medicinal plants [3]. Rumex abyssinicus is a large annual herbaceous plant with generally sagittate leaves, highly branched inflorescence and light brown hazelnuts and locally named in Amharic "mekmako" [4]. It is a medicinal plant widely distributed in the highlands of tropical Africa and distributed throughout North Africa and Ethiopia [5]. Indeed, Rumex abyssinicus is used in traditional medicine to treat several diseases like rheumatism, malaria, typhoid and hepatitis [5] [6]. Members of the genus Rumex have been reported to produce a wide range of secondary metabolites such as anthraquinones [7], flavonoids [8], stilbenoids [9] [10], tannins [11], triterpenoids [12], steroids and saponins [13]. Some of them possess several pharmacological activities such as antioxidant, antitumour, antimicrobial, antiparasitic, antiviral [14]. Previous pharmacological studies carried out on $R$. abyssinicus revealed its antimicrobial activities against Salmonella typhimurium, Listeria monocytogenes (ATCC 29211), Escherichia coli (ATCC25922), Staphylococcus aureus (ATCC 4944) [15] and antiplasmodial activity against chloroquine-sensitive Plasmodium falciparum strain (3D7) [16]. In our continuous search for bioactive secondary metabolites from Cameroonian medicinal plants [17] [18] [19] [20], we report in this paper the isolation, characterization, antibacterial activities and Chemophenetic significance of secondary metabolites from the whole plant of $R$. abyssinicus. 


\section{Materials and Methods}

\subsection{General Experimental Procedures}

Bruker IR-Alpha spectrometer apparatus was used for scanning IR (Infrared) spectroscopy using $\mathrm{KBr}$ pellets. Column chromatography was carried out on silica gel 230 - 400 mesh, Merck (Merck, Darmstadt, Germany), 70 - 230 mesh (Merck) and/or gel permeation on Sephadex LH-20 (Sigma-Aldrich, Munich, Germany). High resolution mass spectra were obtained with QTOF (Quadrupole Time of Flight) Compact Spectrometer (Bruker, Germany) equipped with a HRESI source. The spectrometer was operated in positive and negative modes (mass range: $50-1500$, with a scan rate of $1.00 \mathrm{~Hz}$ ) with automatic gain control to provide high-accuracy mass measurements within $0.4 \mathrm{ppm}$ deviation using $\mathrm{Na}$ formate as calibrant. The following parameters were used for experiments: spray voltage of $4.5 \mathrm{kV}$, capillary temperature of $200^{\circ} \mathrm{C}$. Nitrogen was used as sheath gas (4 L/min). The ${ }^{1} \mathrm{H}$ and ${ }^{13} \mathrm{C}$ Nuclear Magnetic Resonance (NMR) spectra were recorded on Bruker DRX $500 \mathrm{MHz}$ and $600 \mathrm{MHz}$ NMR spectrometers and on a Bruker Avance III 600 and 500 spectrometers equipped with a cryo-platform. 2D NMR experiments were performed using standard Bruker microprograms (XwinNMR version 2.1 software) in deuterated solvents. Chemical shifts ( $\delta$ ) are reported in parts per million (ppm) using the residual solvent signals as secondary reference relatively to TMS (Tetramethylsilane) ( $\delta=0$ ), while the coupling constants $(J$ values) are given in Hertz $(\mathrm{Hz})$. Thin-layer chromatography (TLC) was carried out on Merck pre-coated silica gel $\left(60 \mathrm{~F}_{254}\right)$ aluminium foil (Merck) with detection accomplished by spraying with diluted sulfuric acid $\left(50 \% \mathrm{H}_{2} \mathrm{SO}_{4}\right.$, Riedel-de Haen AG, $95 \%$ - 97\%) followed by heating at $100^{\circ} \mathrm{C}$, or by visual inspection under Ultraviolet (UV) lamp at 254 and $365 \mathrm{~nm}$.

\subsection{Plant Material}

In the present study the whole plant of Rumex abyssinicus was collected in February 2018 in Dschang Western Region of Cameroon, with GPS data of: latitude: N 5 27'5.94828" and longitude: E 10 $3^{\circ} 17.39556 "$. The botanical identification was carried out by Victor Nana, a botanist of the National Herbarium of Cameroon, where a specimen was deposited under the voucher number $\mathrm{N}^{\circ} 50551 / \mathrm{HNC}$.

\subsection{Extraction and Isolation}

The air-dried plant material $(4.5 \mathrm{~kg})$ was powdered and extracted at room temperature with methanol $(3 \times 20 \mathrm{~L}, 72 \mathrm{~h})$ to yield $200 \mathrm{~g}$ of crude methanolic extract after evaporation of solvent under reduced pressure. A part of this crude extract (195 g) was dissolved in water $\left(\mathrm{H}_{2} \mathrm{O}, 300 \mathrm{~mL}\right)$ followed by a liquid-liquid extraction with ethyl acetate (EtOAc, $500 \mathrm{~mL})$ and $n$-butanol $(n-\mathrm{BuOH}, 500 \mathrm{~mL})$ yielding respectively $50 \mathrm{~g}$ and $18 \mathrm{~g}$ after solvents evaporation under vacuum (at $\left.40^{\circ} \mathrm{C}\right)$. A part of the EtOAc fraction $(45 \mathrm{~g}$, i.e $90 \%$ of the mass obtained) was subjected to silica gel column chromatography using $n$-hexane-EtOAc $(95: 5 \rightarrow$ 80:20, v/v) followed by EtOAc- $\mathrm{MeOH}(95: 5 \rightarrow 70: 30, \mathrm{v} / \mathrm{v})$ for a gradient elution. 
Seventy (70) fractions of $400 \mathrm{~mL}$ were collected and combined on the basis of their TLC profiles to give 8 fractions A-H (A: 1 - 3; B: 4 - 10; C: 11 - 22; D: 23 28; E: 29 - 35; F: 36 - 44; G: 45 - 63; H: 64 - 70). Compounds 1 (30.8 mg), 17 (15.5 mg) [21] and 2 (6.8 mg) [22] were obtained after purification of fraction $\mathrm{F}$ (2 g) on silica gel column chromatography using $n$-hexane-EtOAc $(40: 60 \rightarrow$ $00: 100, \mathrm{v} / \mathrm{v})$. Fraction A (4 g) was purified on silica gel column chromatography with $n$-hexane-EtOAc as eluent (95:5, v/v) to yield compounds 9 (10.2 mg) [23], 3 (15 mg) [24] and 4 (17 mg) [25]. Fraction B (5 g) was also purified on silica gel column chromatography eluted with $n$-hexane-EtOAc $(95: 5 \rightarrow 90: 10, \mathrm{v} / \mathrm{v})$ to give a mixture of two compounds (15 and 16) (12.5 mg) [26] and compound 10 (6.4 mg) [27]. Sephadex LH-20 gel column chromatography of fraction C ( $1.9 \mathrm{~g})$ led to two sub-fractions labelled $\mathrm{C}_{1}$ and $\mathrm{C}_{2}$. The purification of the sub-fraction $\mathrm{C}_{1}$ $(500 \mathrm{mg})$ by silica gel column chromatography using $n$-hexane-EtOAc, $(90: 10 \rightarrow$ $80: 20, \mathrm{v} / \mathrm{v})$ as mobile phase afforded to compounds 14 (4.1 mg) [28], 11 (7.6 mg) [27], 12 (8.2 mg) [29] and 13 (15 mg) [30]. Sub-fraction $C_{2}$ (300 mg), was purified on Sephadex LH-20 gel column chromatography using $\mathrm{MeOH}$ as eluent to give compound 6 (40 mg) [25]. Fraction D (3.74 g) was also subjected to Sephadex LH-20 gel column chromatography using isocratic elution with $\mathrm{MeOH}$ to obtain three sub-fractions $\mathrm{D}_{1}, \mathrm{D}_{2}$ and $\mathrm{D}_{3}$. Purification of $\mathrm{D}_{3}(400 \mathrm{mg})$ sub-fraction on silica gel column chromatography with $n$-hexane-EtOAc $(85: 15, \mathrm{v} / \mathrm{v})$ gave compound 5 (11 mg) [31]. Recrystallization of fraction G (5 g) afforded a mixture of two compounds 7 and 8 (10 mg) [32].

\subsection{Methanolysis of Compound 1}

Compound 1 (5.0 mg, $7.3421 \mu \mathrm{Mol})$ was dissolved in 5\% $\mathrm{HCl}-\mathrm{MeOH}(3 \mathrm{~mL})$ $(\mathrm{HCl}, 37 \%$ produce by Fisher Scientific and $\mathrm{MeOH}$ obtained from SOLEVO-Cameroon) and refluxed for $14 \mathrm{~h}$ at $70^{\circ} \mathrm{C}$. The reaction was monitored using TLC analysis. At the end of the reaction, the mixture obtained was extracted with methylene chloride $\left(\mathrm{CH}_{2} \mathrm{Cl}_{2}\right.$ obtained from SOLEVO-Cameroon) after neutralization with dilute potassium carbonate $\left(\mathrm{K}_{2} \mathrm{CO}_{3}\right.$, produce by Fisher Scientific with $99.9 \%$ purity). The organic phase was separated and concentrated to yield $2.1 \mathrm{mg}$.

\subsection{Methodology of Antibacterial Assay}

\subsubsection{Bacteria Strains and Sample Preparation}

The microorganisms used in this study were consisted of four bacterial strains namely: Staphylococcus aureus NR 43300, Shighella flexineri NR 518, Klebsiella, pneumonia ATCC 700603, Escherichia coli ATCC 25922 obtained from our local stocks. They were cultivated in petri dishes containing Mueller Hinton Agar (MHA) followed by an incubation period of 24 hours at $37^{\circ} \mathrm{C}$. Each microorganism was sub-cultured in a new agar plate and incubated in the same above mentioned conditions prior each experiment. Extracts and compounds were weighed and dissolved in different solvents (pyridine, DMSO) for a final of $100 \mathrm{mg} / \mathrm{mL}$ and $20 \mathrm{mg} / \mathrm{mL}$ for extracts and compounds respectively. Positive control Ci- 
profloxacin was prepared at $1 \mathrm{mg} / \mathrm{mL}$. Extracts, compounds and the reference drugs were screened at $1000 \mu \mathrm{g} / \mathrm{mL}, 500 \mu \mathrm{g} / \mathrm{mL}$ and $119 \mu \mathrm{g} / \mathrm{mL}$, respectively.

\subsubsection{Determination of Minimal Inhibitory Concentration (MIC)}

The antimicrobial activity of each product was done as previously described by the CLSI, (2012) using the broth microdilution method in 96-wells microplates. Briefly, $196 \mu \mathrm{L}$ and $190 \mu \mathrm{L}$ of culture media (Nutrient Broth (NB) for extracts and compounds respectively were introduced in plates and $4 \mu \mathrm{L}$ and $10 \mu \mathrm{L}$ of test products were added only in wells belonging to the first line. After a gentle homogenization, serial two-fold dilutions of test products were made by transferring $100 \mu \mathrm{L}$ of the mixture from the first well to the second up to the last. 100 $\mu \mathrm{L}$ of the microbial inoculum standardized at $5.105 \mathrm{UFC} / \mathrm{Ml}$ (With $0.5 \mathrm{McFar}-$ land standard) were respectively introduced in wells. Wells containing the culture medium only served as sterility control, those containing the microorganisms and the culture medium were the Negative control corresponding to one hundred percent growth (100\% growth). Positive control Ciprofloxacin was distinctively screened in same conditions. All the experiments were performed in duplicate and twice. The plates were incubated for 24 hours at $37^{\circ} \mathrm{C} .20$ microliters of resazurin $(0.15 \mathrm{mg} / \mathrm{mL}$ in PBS) were then added in duplicate wells and plates were further incubated for 2 hours. MICs were determined as the lowest concentration in which no visible growth (blue colored wells) was observed in wells after the incubation period. Test concentrations were $1000-15.625 \mu \mathrm{g} / \mathrm{mL}$ and $500-7.875 \mu \mathrm{g} / \mathrm{mL}$ for extracts and compounds respectively.

\section{Results and Discussion}

The structures of compounds 2-17 were determined on the basis of the spectroscopic and mass spectrometric data as bis-(2-ethylhexyl) phthalate (2) [22], Chrysophanol (3) [24], physcion (4), citreorosein (5) [31], emodin (6) [25], chrysophanein (7) and physcionin (8) [32], lupeol (9) [23], 3 $\beta, 28$-dihydroxylup-20(29)-ene (10) [27], 3 $\beta$-dihydroxylup-20(29)-en-28-oic acid (11) [27], oleanolic acid (12) [29], ergosta-6,22-diene-3,5,8-triol (13) [30], stigmastane-3,6-dione (14) [28], $\beta$-sitosterol (15) and stigmasterol (16) [26], stigmasterol 3-O- $\beta$-Dglucoside (17) [21] (Figure 1).

Compound 1 was obtained as white powder in $n$-hexane-EtOAc $(30: 70, \mathrm{v} / \mathrm{v})$. Its molecular formula $\mathrm{C}_{42} \mathrm{H}_{83} \mathrm{NO}_{5}$ was determined on the basis of its HR-TOFESIMS spectrum which showed a sodium adduct peak $[\mathrm{M}+\mathrm{Na}]^{+}$at $\mathrm{m} / \mathrm{z} 704.6133$ (calcd. $\mathrm{C}_{42} \mathrm{H}_{83} \mathrm{NO}_{5} \mathrm{Na}$, 704.6163), indicating two degrees of insaturation. The IR spectrum displayed characteristic vibration bands for hydroxy group $\left(3315 \mathrm{~cm}^{-1}\right)$, olefinic group $\left(1626 \mathrm{~cm}^{-1}\right)$ and amide carbonyl group $\left(1655 \mathrm{~cm}^{-1}\right)$. The ${ }^{1} \mathrm{H}$ NMR spectrum indicated signals of $(-\mathrm{NH})$ at $\delta_{\mathrm{H}} 8.61(1 \mathrm{H}, d, J=9.1 \mathrm{~Hz})$, oxygenated methylene at $\delta_{\mathrm{H}} 4.54(1 \mathrm{H}, m, \mathrm{H}-1 \mathrm{a})$ and $4.45(1 \mathrm{H}, m, \mathrm{H}-1 \mathrm{~b})$, three oxymethine protons at $\delta_{\mathrm{H}} 4.65\left(1 \mathrm{H}, m, \mathrm{H}-2^{\prime}\right), 4.39(1 \mathrm{H}, m, \mathrm{H}-3)$ and $4.32(1 \mathrm{H}, m, \mathrm{H}-4)$, 

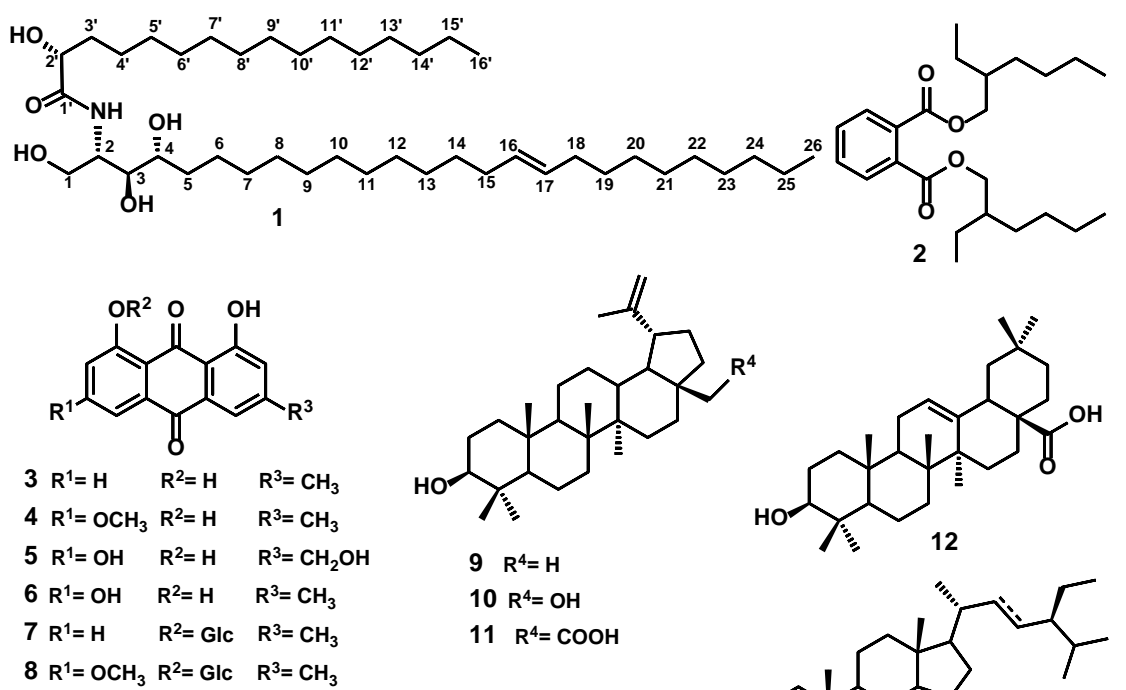

$8 \mathrm{R}^{1}=\mathrm{OCH}_{3} \mathrm{R}^{2}=\mathrm{GlC} \quad \mathrm{R}^{3}=\mathrm{CH}_{3}$
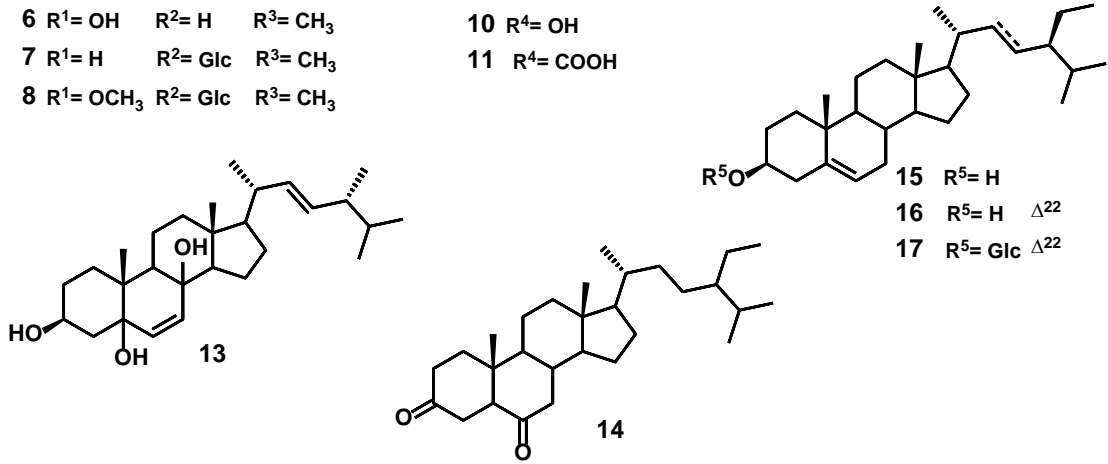

Figure 1. Structures of compounds 1-17 isolated from EtOAc soluble fraction of crude extract of Rumex abyssinicus.

two terminal methyls at $\delta_{\mathrm{H}} 0.88(6 \mathrm{H}, t, J=7.0 \mathrm{~Hz}, \mathrm{H}-16$ and $\mathrm{H}-26)$. A set of signals appearing as broad in the range of $\delta_{\mathrm{H}} 1.52-1.20$ corresponding to the methylenes $\left(-\mathrm{CH}_{2}-\right)$ associated to the two long chains, and a downfield signal at $\delta_{\mathrm{H}}$ $5.14(1 \mathrm{H}, m, \mathrm{H}-2)$ assigned to the aminomethine $\mathrm{H}-2$ of sphingosine were also observed [33]. All the above spectral data (Table 1) revealed that compound 1 was a phytosphingosine type sphingolipid [34]. In addition, its ${ }^{1} \mathrm{H}$ NMR spectrum exhibited two olefinic protons at $\delta_{\mathrm{H}} 5.57(1 \mathrm{H}, \mathrm{m}, \mathrm{H}-16)$ and $5.51(1 \mathrm{H}, \mathrm{m}$, $\mathrm{H}-17)$.

A concomitant analysis of its ${ }^{13} \mathrm{C}-\mathrm{NMR}$, DEPT and HSQC spectra was in agreement with the ${ }^{1} \mathrm{H}$-NMR spectrum and allowed to identify an amide carbonyl carbon at $\delta_{\mathrm{C}} 175.5\left(\mathrm{C}-1^{\prime}\right)$, two olefinic methine carbons resonated at $\delta_{\mathrm{C}}$ $131.2(\mathrm{C}-16)$ and $131.1(\mathrm{C}-17)$, an oxymethylene carbon resonated at $\delta_{\mathrm{C}} 62.4$ $(\mathrm{C}-1)$, four $\mathrm{sp}^{3}$ methine carbon signals in which, three oxymethines at $\delta_{\mathrm{C}} 77.2$ (C-3), $73.3(\mathrm{C}-4), 72.8\left(\mathrm{C}-2^{\prime}\right)$, and an aminomethine at $\delta_{\mathrm{C}} 53.2(\mathrm{C}-2)$. It also revealed aliphatic methylenic carbons in the range of $\delta_{\mathrm{C}} 36.1-23.2$, and two terminal methyl carbons at $\delta_{\mathrm{C}} 14.6 \mathrm{ppm}$. The trans $(E)$ configuration of the double bond was confirmed by the chemical shifts of the allylic carbons at $\delta_{\mathrm{C}} 33.7$ (C-15) and 33.6 (C-18) [35] [36]. The correlation observed on its COSY ${ }^{1} \mathrm{H}-{ }^{1} \mathrm{H}$ (Figure 2) spectrum between protons: $\mathrm{H}-1 \mathrm{a} / \mathrm{H}-1 \mathrm{~b}$ and $\mathrm{H}-2, \mathrm{H}-2$ and $\mathrm{H}-3, \mathrm{H}-3$ and $\mathrm{H}-4$ allowed us to identify the position of four hydroxy groups. This was further confirmed by the HMBC correlations between the protons at $\delta_{\mathrm{H}} 4.54$ 
Table $1 .{ }^{1} \mathrm{H}(600 \mathrm{MHz})$ and ${ }^{13} \mathrm{C}(150 \mathrm{MHz})$ NMR data of rumexamide (1) in pyridine- $d_{5}$ ( $\delta$ in ppm, $J$ in $\mathrm{Hz}$, TMS as internal standard).

\begin{tabular}{|c|c|c|c|c|}
\hline Position & $\delta_{H}(\mathrm{nH}$, mult; $J$ in $\mathrm{Hz})$ & $\delta_{\mathrm{C}}$ & (mult) & HMBC \\
\hline \multicolumn{5}{|c|}{ Long chain base } \\
\hline $\mathrm{NH}$ & $8.61(1 \mathrm{H}, d, J=9.1)$ & - & & - \\
\hline $1 \mathrm{a}, 1 \mathrm{~b}$ & $\begin{array}{l}4.54(1 \mathrm{H}, m) \\
4.45(1 \mathrm{H}, m)\end{array}$ & 62.4 & $\left(\mathrm{CH}_{2}\right)$ & $2 ; 3$ \\
\hline 2 & $5.14(1 \mathrm{H}, m)$ & 53.2 & $(\mathrm{CH})$ & $1 ; 1 ; 3$ \\
\hline 3 & $4.39(1 \mathrm{H}, \mathrm{m})$ & 77.2 & $(\mathrm{CH})$ & $1 ; 2 ; 4 ; 5$ \\
\hline 4 & $4.32(1 \mathrm{H}, m)$ & 73.3 & $(\mathrm{CH})$ & 5 \\
\hline 5 & $2.00(2 \mathrm{H}, m)$ & 34.2 & $\left(\mathrm{CH}_{2}\right)$ & - \\
\hline $6-14$ & $1.52-1.20(18 \mathrm{H}$, brs $)$ & $32.5-27.0$ & $\left(\mathrm{CH}_{2}\right)_{9}$ & - \\
\hline 15 & $2.19(2 \mathrm{H}, m)$ & 33.7 & $\left(\mathrm{CH}_{2}\right)$ & 16 \\
\hline 16 & $5.57(1 \mathrm{H}, m)$ & 131.2 & $(\mathrm{CH})$ & 15 \\
\hline 17 & $5.51(1 \mathrm{H}, m)$ & 131.1 & $(\mathrm{CH})$ & 18 \\
\hline 18 & $2.02(2 \mathrm{H}, m)$ & 33.6 & $\left(\mathrm{CH}_{2}\right)$ & 17 \\
\hline $19-24$ & $1.52-1.20(10 \mathrm{H}$, brs $)$ & $32.5-27.0$ & $\left(\mathrm{CH}_{2}\right)_{5}$ & \\
\hline 25 & $1.26(1 \mathrm{H}, m)$ & 23.3 & $\left(\mathrm{CH}_{2}\right)$ & - \\
\hline 26 & $0.88(3 \mathrm{H}, t, J=7.0)$ & 14.6 & $\left(\mathrm{CH}_{3}\right)$ & 25 \\
\hline \multicolumn{5}{|c|}{$\mathrm{N}$-acyl moiety } \\
\hline $1^{\prime}$ & - & 175.5 & (C) & - \\
\hline $2^{\prime}$ & $4.65(2 \mathrm{H}, d t, J=7.7,3.7)$ & 72.8 & $\left(\mathrm{CH}_{2}\right)$ & \\
\hline $3^{\prime}$ & $\begin{array}{l}2.26(1 \mathrm{H}, m) \\
2.06(1 \mathrm{H}, m)\end{array}$ & 36.1 & $\left(\mathrm{CH}_{2}\right)$ & $\begin{array}{l}- \\
2^{\prime}\end{array}$ \\
\hline $4^{\prime}-14^{\prime}$ & $1.52-1.20(22 \mathrm{H}$, brs $)$ & $32.5-27.0$ & $\left(\mathrm{CH}_{2}\right)_{11}$ & - \\
\hline $15^{\prime}$ & $1.26(1 \mathrm{H}, m)$ & 23.3 & $\left(\mathrm{CH}_{2}\right)$ & - \\
\hline $16^{\prime}$ & $0.88(3 \mathrm{H}, t, J=7.0)$ & 14.6 & $\left(\mathrm{CH}_{3}\right)$ & $15^{\prime}$ \\
\hline
\end{tabular}

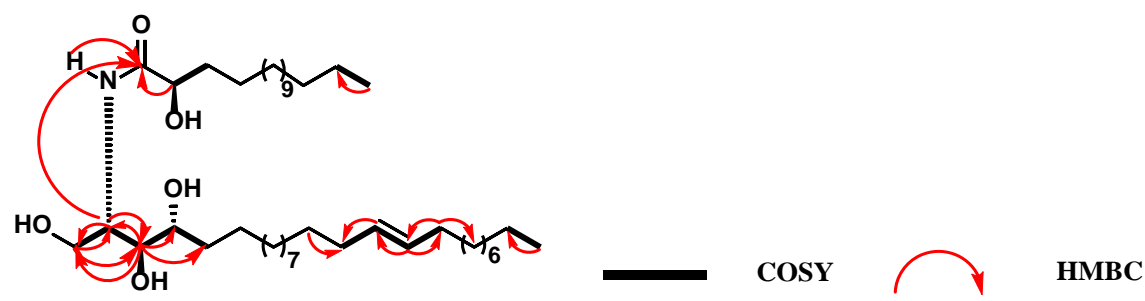

Figure 2. Selected COSY and HMBC correlations for compound 1.

$(\mathrm{H}-1 \mathrm{a}), 4.45(\mathrm{H}-1 \mathrm{~b})$ and the carbons at $\delta_{\mathrm{C}}: 77.2(\mathrm{C}-3), 53.2(\mathrm{C}-2)$; between the proton at $\delta_{\mathrm{H}} 4.39(\mathrm{H}-3)$ and the carbons at $\delta_{\mathrm{C}}: 73.3(\mathrm{C}-4), 62.3(\mathrm{C}-1), 53.2(\mathrm{C}-2)$, $34.2(\mathrm{C}-5)$ and between the proton of hydroxymethine at $\delta_{\mathrm{H}} 4.65\left(\mathrm{H}-2^{\prime}\right)$ and 
carbonyl at $\delta_{\mathrm{C}} 175.5\left(\mathrm{C}-1^{\prime}\right)$. In addition, correlation observed between the proton of aminomethine at $\delta_{\mathrm{H}} 5.14(\mathrm{H}-2)$ and carbons at $\delta_{\mathrm{C}} 77.2(\mathrm{C}-3), 62.4(\mathrm{C}-1)$ and $175.5\left(\mathrm{C}-1^{\prime}\right)$ allowed to conclude that, the two chains (acid and basic) are linked. The absolute configuration of the stereocenters C-2, C-3, C-4 and C-2', were assigned to be $2 R, 3 S, 4 R, 2^{\prime} R$ on the basis of their carbon chemical shifts which are very close to those of vernoguinamide [37].

The length of the acid chain (ALC) was determined using the ESI (-) mass spectrum where characteristic fragment ions are observed at $m / z .255\left[\mathrm{CH}_{3}\left(\mathrm{CH}_{2}\right)_{13}\right.$ $\mathrm{CH}(\mathrm{OH}) \mathrm{CO}]^{-}, 227\left[\mathrm{CH}_{3}\left(\mathrm{CH}_{2}\right)_{13} \mathrm{CH}(\mathrm{OH})\right]^{-}, 199\left[\mathrm{HNCOCH}(\mathrm{OH})\left(\mathrm{CH}_{2}\right)_{9}\right]^{-}, 170$ $\left[\mathrm{CH}_{3}\left(\mathrm{CH}_{2}\right)_{11}+\mathrm{H}\right]^{-}, 156\left[\mathrm{CH}_{3}\left(\mathrm{CH}_{2}\right)_{10}+\mathrm{H}\right]^{-}$. Concerning the basic chain, the length was deduced from the mass of the entire molecule by subtracting that of the acid chain. Thus, said basic chain has 426 for mass, corresponding to $\left[\mathrm{CH}_{3}\left(\mathrm{CH}_{2}\right)_{8} \mathrm{CH}=\mathrm{CH}\left(\mathrm{CH}_{2}\right)_{11} \mathrm{CH}(\mathrm{OH}) \mathrm{CH}(\mathrm{OH}) \mathrm{CH}(\mathrm{NH}) \mathrm{CH}_{2}(\mathrm{OH})\right]$ having a double bond. Note that this number of carbon atoms of the acid and basic chains was further confirmed by the methanolysis of said compound which led to a fatty acid methyl ester unit (FAME; 1a) and a long chain phytosphingosine unit (LCB; 1b) (Scheme 1).

The LC-MS analysis of the organic phase showed that, the methanolysis of compound 1 led to the formation of methyl $(R)$ 2-hydroxyhexadecanoate (1a), $\left(\mathrm{m} / z 309.1[\mathrm{M}+\mathrm{Na}]^{-}\right)$and $(2 S, 3 S, 4 R, 16 E)$-2-aminohexacos-16-ene-1,3,4-triol (1b), $\left(\mathrm{m} / z 468.5\left[\mathrm{M}+\mathrm{CH}_{3} \mathrm{CN}\right]^{+}\right.$and $\left.\mathrm{m} / z 451.3[\mathrm{M}+\mathrm{H}+\mathrm{Na}]^{+}\right)$(Scheme 1) indicating 16 carbon atoms for acid chain (1a) and 26 carbon atoms with one double bond for basic chain (1b). The position of the double bond was deduced from its ESI (+) mass spectrum on which ions fragments are observed at $m / z 554\left[\mathrm{M}-\mathrm{C}_{9} \mathrm{H}_{19}\right]^{+}$ and $m / z 152\left[\mathrm{C}_{11} \mathrm{H}_{21}-\mathrm{H}\right]^{+}$(Figure 3 ). Accordingly, the structure of compound 1 was established as $(R)-2$-hydroxy- $N$ - $[(2 S, 3 S, 4 R, 16 E)$-1,3,4-trihydroxyhexacos-16en-2-yl] hexadecanamide, trivially named rumexamide.

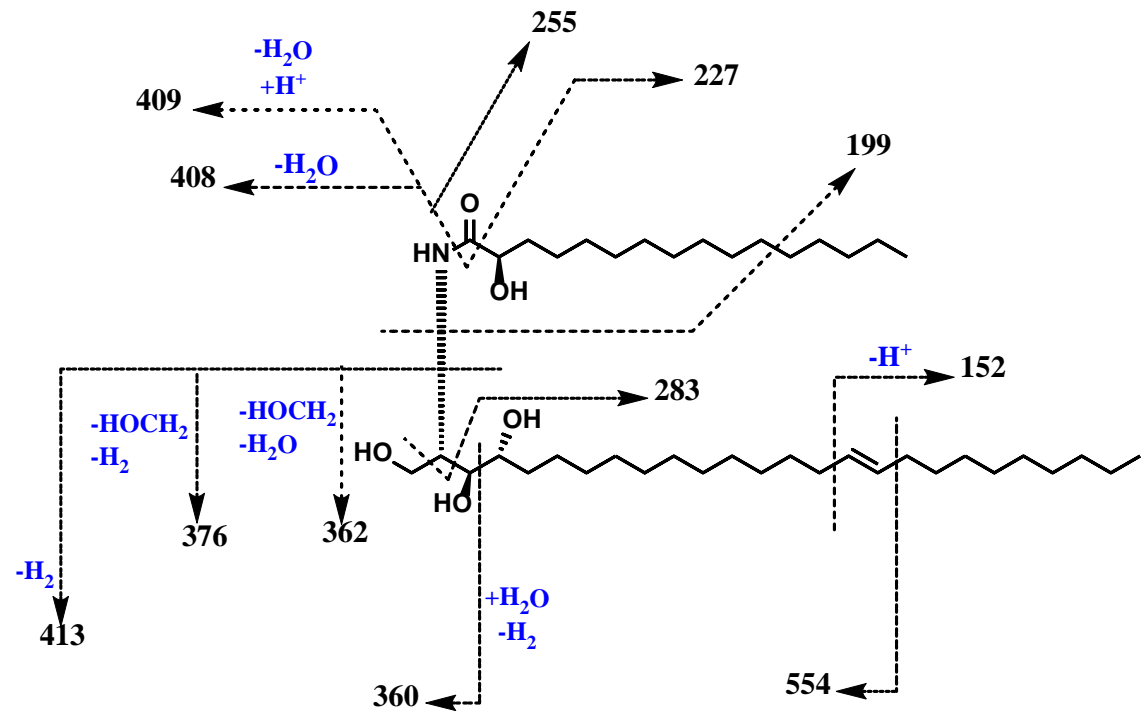

Figure 3. Mass fragmentation pattern for compound 1 . 


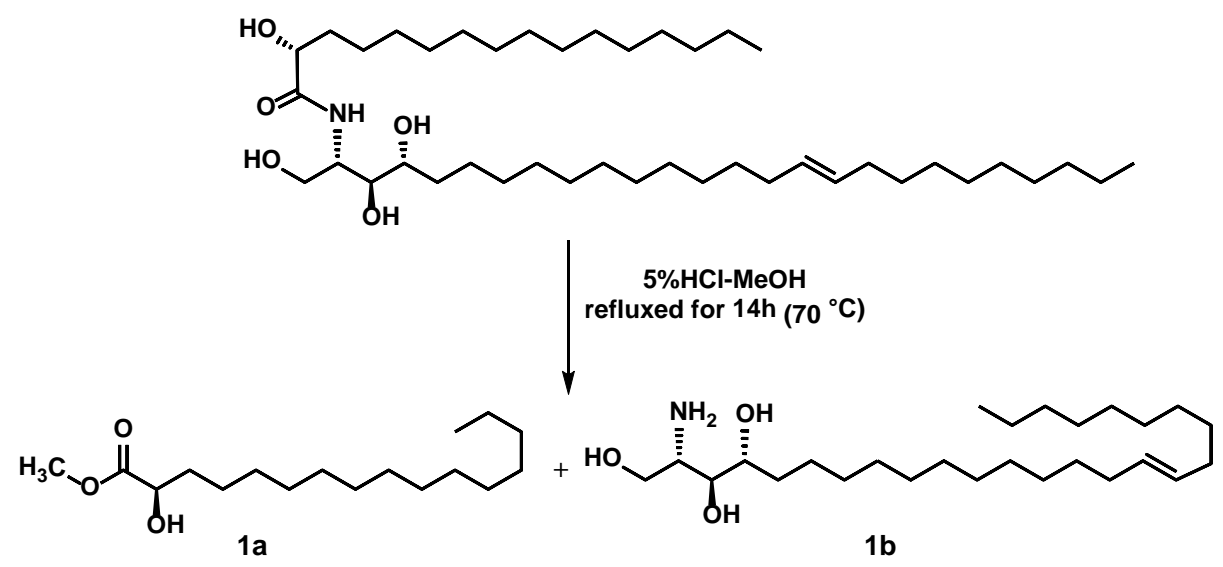

Scheme 1. Methanolysis of 1.

The antibacterial activities of $\mathrm{MeOH}, \mathrm{EtOAc}$ and $n-\mathrm{BuOH}$ extracts as well as compounds $1,9,11,12,15+16$ and 17 against pathogenic bacteria are presented in Table 2. In general, the test samples demonstrated weak variation degrees of inhibitory activity against the bacteria. The antibacterial activity of the plant extract can be classified as significant (MIC $<100 \mu \mathrm{g} / \mathrm{mL}$ ), moderate $(100<$ MIC $\leq 625 \mu \mathrm{g} / \mathrm{mL}$ ) and weak (MIC $>625 \mu \mathrm{g} / \mathrm{mL}$ ) whereas antimicrobial activity of pure compound can be classified as significant (MIC $<10 \mu \mathrm{g} / \mathrm{mL}$ ), moderate $(10<$ MIC $\leq 100 \mu \mathrm{g} / \mathrm{mL})$ and weak (MIC $>100 \mu \mathrm{g} / \mathrm{mL}$ ) [38]. Base on this, extract were not active (MIC $>1000 \mu \mathrm{g} / \mathrm{mL}$ ) while compounds were weakly or not active (MIC $\geq 500 \mu \mathrm{g} / \mathrm{mL}$ ) against all bacteria strains.

\section{Spectroscopic Data of Compounds 1-17}

Bis-(2-ethylhexyl) phthalate (2) - White powder; $\left(\mathrm{C}_{24} \mathrm{H}_{38} \mathrm{O}_{4}\right) ;{ }^{1} \mathrm{H}-\mathrm{NMR}$ (400 $\left.\mathrm{MHz}, \mathrm{CDCl}_{3}\right): \delta_{\mathrm{H}}: 7.70(d d, 5.7,3.3 \mathrm{~Hz}, \mathrm{H}-3), 7.52(d d, 5.7,3.3 \mathrm{~Hz}, \mathrm{H}-4), 4.22(\mathrm{~m}$, H-1 ), 1.68 ( $\left.p, 6.1 \mathrm{~Hz}, \mathrm{H}-2^{\prime}\right), 1.41(m, \mathrm{H}-7), 1.34(m, \mathrm{H}-3), 1.32(m, \mathrm{H}-4), 1.31$ $(m, \mathrm{H}-5), 0.92(m, \mathrm{H}-8), 0.89(m, \mathrm{H}-6) ;{ }^{13} \mathrm{C}-\mathrm{NMR}\left(100 \mathrm{MHz}, \mathrm{CDCl}_{3}\right) \delta_{\mathrm{C}}: 167.9$ (C-1), 132.6 (C-2), 130.9 (C-4), 128.9 (C-3), 68.3 (C-1), 38.9 (C-2), 30.5 (C-3), 29.1 (C-4), 23.9 (C-7) ), 23.1 (C-5), 14.2 (C-6), 11.1 (C-8).

Chrysophanol (3)-Yellow powder; $\left(\mathrm{C}_{15} \mathrm{H}_{10} \mathrm{O}_{4}\right):{ }^{1} \mathrm{H}-\mathrm{NMR}\left(600 \mathrm{MHz}, \mathrm{CDCl}_{3}\right)$ $\delta_{\mathrm{H}}: 12.08(s, 1-\mathrm{OH}), 11.97(s, 8-\mathrm{OH}) 7.84(d, 7.5 \mathrm{~Hz}, \mathrm{H}-5), 7.77$ (br $\left.s, \mathrm{H}-6\right), 7.69$ (br $s, \mathrm{H}-4), 7.30$ (d, 8.4Hz, H-7), 7.12 (br $s, \mathrm{H}-2), 2.48\left(s,-\mathrm{CH}_{3}\right) ;{ }^{13} \mathrm{C}-\mathrm{NMR}(150$ $\left.\mathrm{MHz}, \mathrm{CDCl}_{3}\right) \delta_{\mathrm{C}}: 192.5$ (C-9), 182.1 (C-10), 162.7 (C-1), 162.4 (C-8), 149.3 (C-3), 137.0 (C-6), 133.6 (C-11), 133.2 (C-14), 124.5 (C-7), 124.3 (C-2), 121.3 (C-4), 119.9 (C-5), 115.8 (C-12), 113.7 (C-13), $22.4\left(-\mathrm{CH}_{3}\right)$.

Physcion (4)-Yellow powder; $\left(\mathrm{C}_{16} \mathrm{H}_{12} \mathrm{O}_{5}\right):{ }^{1} \mathrm{H}-\mathrm{NMR}\left(600 \mathrm{MHz}, \mathrm{CDCl}_{3}\right) \delta_{\mathrm{H}}$ : $12.34(s, 1-\mathrm{OH}), 12.15$ ( $s, 8-\mathrm{OH}), 7.65$ (br s, H-5), 7.39 (d, $2.5 \mathrm{~Hz}, \mathrm{H}-4), 7.10$ (br $s, \mathrm{H}-7), 6.70(d, 2.5 \mathrm{~Hz}, \mathrm{H}-2), 3.95\left(s, \mathrm{OCH}_{3}\right), 2.46\left(s,-\mathrm{CH}_{3}\right) ;{ }^{13} \mathrm{C}-\mathrm{NMR}(150$ $\left.\mathrm{MHz}, \mathrm{CDCl}_{3}\right) \delta_{\mathrm{C}}: 190.8(\mathrm{C}-9), 182.1$ (C-10), 166.6 (C-3), 165.2 (C-1), 162.5 (C-8), 148.5 (C-6), 135.2 (C-14), 133.2 (C-11), 124.6 (C-7), 121.4 (C-5), 113.7 (C-12), 110.3 (C-13), $108.3(\mathrm{C}-4), 106.8(\mathrm{C}-2), 56.1\left(-\mathrm{OCH}_{3}\right), 22.2\left(-\mathrm{CH}_{3}\right)$. 
Table 2. Antimicrobial activities of extracts, some isolated compounds and ciprofloxacin.

\begin{tabular}{|c|c|c|c|c|c|}
\hline \multirow{2}{*}{ Samples } & \multirow{2}{*}{$\begin{array}{l}\text { Parameters } \\
(\mu \mathrm{g} / \mathrm{mL})\end{array}$} & \multicolumn{4}{|c|}{ Bacteria strains } \\
\hline & & $S a$ & $S f$ & $K p$ & $E c$ \\
\hline EtOAc extract & MIC & $>1000$ & $>1000$ & $>1000$ & $>1000$ \\
\hline$n-\mathrm{BuOH}$ extract & MIC & $>1000$ & $>1000$ & $>1000$ & $>1000$ \\
\hline $\mathrm{MeOH}$ extract & MIC & $>1000$ & $>1000$ & $>1000$ & $>1000$ \\
\hline 1 & MIC & $>500$ & $500 \pm 000$ & $>500$ & $>500$ \\
\hline 9 & MIC & $>500$ & $>500$ & $>500$ & $>500$ \\
\hline 11 & MIC & $>500$ & $>500$ & $>500$ & $>500$ \\
\hline 12 & MIC & $>500$ & $>500$ & $>500$ & $>500$ \\
\hline $15+16$ & MIC & $>500$ & $>500$ & $>500$ & $>500$ \\
\hline 17 & MIC & $>500$ & $>500$ & $>500$ & $>500$ \\
\hline Ciprofloxacin & MIC & $0.232 \pm 000$ & $0.232 \pm 000$ & $0.464 \pm 000$ & $0.232 \pm 000$ \\
\hline
\end{tabular}

Sa: Staphylococcus aureus ATCC 43300; Sf. Shighella flexineri NR 518; Kp: Klebsiella pneumonia ATCC 700603; Ec. Escherichia coli ATCC 25922; MIC: Minimum Inhibitory Concentration; Ciprofloxacin: reference drug.

Citreorosein (5)-Red powder; $\left(\mathrm{C}_{15} \mathrm{H}_{10} \mathrm{O}_{6}\right)$ : ${ }^{1} \mathrm{H}-\mathrm{NMR}\left(600 \mathrm{MHz}, \mathrm{CD}_{3} \mathrm{OD}\right)$ $\delta_{\mathrm{H}}: 7.28$ (br s, H-2), 7.75 (br s, H-4), 7.20 (br s, H-5), 6.54 (br $\left.s, \mathrm{H}-7\right), 4.70$ ( $s$, - $\mathrm{OCH}_{2}$ ); ${ }^{13} \mathrm{C}-\mathrm{NMR}\left(150 \mathrm{MHz}, \mathrm{CD}_{3} \mathrm{OD}\right) \delta_{\mathrm{C}}: 191.5(\mathrm{C}-9), 183.4(\mathrm{C}-10), 169.1(\mathrm{C}-8)$, 166.7 (C-6), 163.7 (C-1), 152.9 (C-3), 136.9 (C-11), 135.0 (C-14), 122.2 (C-2), 118.4 (C-4), 115.9 (C-13), 111.1 (C-5), 109.2 (C-7), 108.5 (C-12), 64.1 (-OCH $\left.2^{-}\right)$.

Emodin (6)-Red powder; $\left(\mathrm{C}_{15} \mathrm{H}_{10} \mathrm{O}_{5}\right):{ }^{1} \mathrm{H}-\mathrm{NMR}\left(600 \mathrm{MHz}, \mathrm{DMSO}-d_{6}\right) \delta_{\mathrm{H}}$ : $12.1(s, 3-\mathrm{OH}), 12.0(s, 8-\mathrm{OH}) 7.48(d, 0.7 \mathrm{~Hz}, \mathrm{H}-5), 7.16(d, 0.7 \mathrm{~Hz}, \mathrm{H}-7) 7.11(d$, $2.4 \mathrm{~Hz}, \mathrm{H}-4) 6.59(d, 2.4 \mathrm{~Hz}, \mathrm{H}-2), 2.41\left(s,-\mathrm{CH}_{3}\right) ;{ }^{13} \mathrm{C}-\mathrm{NMR}\left(150 \mathrm{MHz}, \mathrm{DMSO}-d_{6}\right)$ $\delta_{\mathrm{C}}$ : 190.2 (C-9), 181.9 (C-10), 166.1 (C-1), 164.9 (C-3), 161.9 (C-8), 148.7 (C-6), 135.6 (C-14), 133.3 (C-11), 124.6 (C-7), 120.9 (C-5), 113.8 (C-12), 109.4 (C-13), 109.3 (C-4), 108.4 (C-2), $21.9\left(-\mathrm{CH}_{3}\right)$.

Chrysophanein (7)-Yellow powder; $\left(\mathrm{C}_{21} \mathrm{H}_{20} \mathrm{O}_{9}\right)$ : ${ }^{1} \mathrm{H}-\mathrm{NMR}(600 \mathrm{MHz}$, DMSO$\left.d_{6}\right) \delta_{\mathrm{H}}: 13.1(s, 1-\mathrm{OH}), 7.88(m, \mathrm{H}-5), 7.86(m, \mathrm{H}-6) 7.71(d ; 7.9 \mathrm{~Hz}, \mathrm{H}-7) 7.51(b r$ $s, \mathrm{H}-4) 7.21$ (br s, H-2) 5.20 - 3.10 (Glu), $2.44\left(s, 3-\mathrm{CH}_{3}\right) ;{ }^{13} \mathrm{C}-\mathrm{NMR}(150 \mathrm{MHz}$, DMSO- $\left.d_{6}\right) \delta_{\mathrm{C}}$ : $188.0(\mathrm{C}-9), 182.6$ (C-10), $162.2(\mathrm{C}-1), 158.7$ (C-8), 148.1 (C-3), 136.4 (C-6), 135.2 (C-11), 132.6 (C-14), 124,5 (C-2), 122.9 (C-7), 121.0 (C-5), 119.8 (C-4), 115.3 (C-12), 115.2 (C-13), 101.0 (C-1'), 77.8 (C-5), 77.0 (C-3), 73.7 (C-2), 70.0 (C-4), $61.1(\mathrm{C}-6), 21,9\left(-\mathrm{CH}_{3}\right)$.

Physcionin (8)-Yellow powder; $\left(\mathrm{C}_{22} \mathrm{H}_{22} \mathrm{O}_{10}\right):{ }^{1} \mathrm{H}-\mathrm{NMR}\left(600 \mathrm{MHz}, \mathrm{DMSO}-d_{6}\right)$ $\delta_{\mathrm{H}}: 12.8(s, 1-\mathrm{OH}), 7.50$ (br s, H-4), 7.37 (d, 2.3Hz, H-5) 7.19 (d, $\left.2.3 \mathrm{~Hz}, \mathrm{H}-7\right)$ 7.18 (br s, H-2), 5.20 - 3.10 (Glu), $3.97\left(s,-\mathrm{OCH}_{3}\right), 2.42\left(s,-\mathrm{CH}_{3}\right) ;{ }^{13} \mathrm{C}-\mathrm{NMR}(150$ $\left.\mathrm{MHz}, \mathrm{DMSO}-d_{6}\right) \delta_{\mathrm{C}}: 186.9$ (C-9), 182.4 (C-10), 165.2 (C-6), 162.1 (C-1), 161.2 (C-8), 147.6 (C-3), 135.1 (C-11), 132.5 (C-14), 124.7 (C-2), 119.7 (C-4), 114.96 (C-13), 114.95 (C-12), 107.9 (C-7), 106.9 (C-5), 101.1 (C-1), 77.9 (C-5), 77.1 (C-3), 73.8 (C-2), 70.3 (C-4), $61.3(\mathrm{C}-6), 56.6\left(-\mathrm{OCH}_{3}\right)$ 21,8 (- $\left.\mathrm{CH}_{3}\right)$.

Lupeol (9)-White powder; $\left(\mathrm{C}_{30} \mathrm{H}_{50} \mathrm{O}\right):{ }^{1} \mathrm{H}-\mathrm{NMR}\left(300 \mathrm{MHz}, \mathrm{CDCl}_{3}\right) \delta_{\mathrm{H}}: 4.73$ 
( $s, \mathrm{H}-29 \mathrm{~b}), 4.62$ ( $s, \mathrm{H}-29 \mathrm{a}), 3.23$ ( $m, \mathrm{H}-3), 2.04$ ( $m, \mathrm{H}-19), 1.73\left(s, 30-\mathrm{CH}_{3}\right), 1.07$ $\left(s, 26-\mathrm{CH}_{3}\right), 1.01\left(s, 23-\mathrm{CH}_{3}\right), 0.99\left(s, 27-\mathrm{CH}_{3}\right), 0.87\left(s, 25-\mathrm{CH}_{3}\right), 0.81\left(s, 24-\mathrm{CH}_{3}\right)$, $0.69(m, \mathrm{H}-5) ;{ }^{13} \mathrm{C}-\mathrm{NMR}\left(75 \mathrm{MHz}, \mathrm{CDCl}_{3}\right) \delta_{\mathrm{C}}: 18.0$ (C-28), 150.6 (C-20), 109.9 (C-29), 79.0 (C-3), 42.8 (C-17), 55.3 (C-5), 50.5 (C-9), 48.3 (C-18), 48.0 (C-19), 43.0 (C-14), 40.6 (C-8), 38.9 (C-4), 38.7 (C-1), 38.1 (C-13), 37.2 (C-10), 40.8 (C-22), 34.8 (C-7), 35.2 (C-16), 28.0 (C-15), 29.9 (C-21), 27.5 (C-23), 27.4 (C-2), 25.2 (C-12), 21.0 (C-11), 18.4 (C-6), 19.3 (C-30), 16.0 (C-25), 16.2 (C-26), 15.4 (C-24), 14.6 (C-27).

3 $\beta$,28-dihydroxylup-20(29)-ene (10)-White powder; $\left(\mathrm{C}_{30} \mathrm{H}_{50} \mathrm{O}_{2}\right):{ }^{1} \mathrm{H}-\mathrm{NMR}$ $\left(300 \mathrm{MHz}, \mathrm{CDCl}_{3}\right) \delta_{\mathrm{H}}: 4.73(s, \mathrm{H}-29 \mathrm{~b}), 4.63(s, \mathrm{H}-29 \mathrm{a}), 3.85(m, \mathrm{H}-28), 3.36(m$, $\mathrm{H}-3), 1.73\left(s, 30-\mathrm{CH}_{3}\right), 1.07\left(s, 27-\mathrm{CH}_{3}\right), 1.03\left(s, 26-\mathrm{CH}_{3}\right), 1.00\left(s, 23-\mathrm{CH}_{3}\right), 0.87$ $\left(s, 25-\mathrm{CH}_{3}\right), 0.75\left(s, 24-\mathrm{CH}_{3}\right) ;{ }^{13} \mathrm{C}-\mathrm{NMR}\left(75 \mathrm{MHz}, \mathrm{CDCl}_{3}\right) \delta_{\mathrm{C}}: 38.7(\mathrm{C}-1), 27.4$ (C-2), 79.0 (C-3), 39.0 (C-4), 55.3 (C-5), 18.3 (C-6), 34.3 (C-7), 40.9 (C-8), 50.4 (C-9), 37.4 (C-10), 20.7 (C-11), 25.3 (C-12), 37.2 (C-13), 42.7 (C-14), 27.1 (C-15), 29.2 (C-16), 47.8 (C-17), 47.8 (C-18), 48.8 (C-19), 150.5 (C-20), 29.7 (C-21), 34.0 (C-22), 28.0 (C-23), 15.2 (C-24), 16.1 (C-25), 15.5 (C-26), 14.9 (C-27), 60.5 (C-28), 109.7 (C-29), 19.0 (C-30).

$3 \beta$-dihydroxylup-20(29)-en-28-oic acid (11)-White powder; $\left(\mathrm{C}_{30} \mathrm{H}_{48} \mathrm{O}_{3}\right)$ : ${ }^{1} \mathrm{H}-\mathrm{NMR}\left(500 \mathrm{MHz}\right.$, Methanol- $\left.d_{5}\right) \delta_{\mathrm{H}}: 4.73$ ( $\left.s, \mathrm{H}-29 \mathrm{~b}\right), 4.61$ ( $\left.s, \mathrm{H}-29 \mathrm{a}\right), 3.14$ (dd, $11.4 \mathrm{~Hz} / 4.7 \mathrm{~Hz}, \mathrm{H}-3), 3.04$ (m, H-19), 2.32 (m, H-13), 2.25 (m, H-16), 1.95 (m, $\mathrm{H}-15 \mathrm{a}), 1.91$ ( $m, \mathrm{H}-22), 1.74$ ( $m, \mathrm{H}-12), 1.71\left(s, 30-\mathrm{CH}_{3}\right), 1.65$ (m, H-18), 1.62 ( $m, \mathrm{H}-1), 1.59$ ( $m, \mathrm{H}-2), 1.56$ ( $m, \mathrm{H}-6), 1.49-1.44$ ( $m, \mathrm{H}-11), 1.40$ ( $m, \mathrm{H} 15 \mathrm{~b}), 1.45$ 1.39 ( $m, \mathrm{H}-7), 1.34$ ( $m, \mathrm{H}-9), 1.19$ ( $m, \mathrm{H}-21), 1.02\left(s, 27-\mathrm{CH}_{3}\right), 0.99\left(s, 26-\mathrm{CH}_{3}\right)$, $0.97\left(s, 23-\mathrm{CH}_{3}\right), 0.88\left(s, 25-\mathrm{CH}_{3}\right), 0.77\left(s, 24-\mathrm{CH}_{3}\right), 0.73(m, \mathrm{H}-5) ;{ }^{13} \mathrm{C}-\mathrm{NMR}$ (125 MHz, Methanol- $d_{5}$ ) $\delta_{\mathrm{C}}: 178.7$ (C-28), 150.6 (C-20), 108.8 (C-29), 78.3 (C-3), 56.1 (C-17), 55.5 (C-5), 50.6 (C-9), 49.0 (C-18), 47.2 (C-19), 42.2 (C-14), 40.6 (C-8), 38.7 (C-4), 38.6 (C-1), 38.3 (C-13), 36.9 (C-10), 36.7 (C-22), 34.2 (C-7), 31.9 (C-16), 30.3 (C-15), 29.4 (C-21), 27.2 (C-23), 26.6 (C-2), 25.5 (C-12), 20.7 (C-11), 18.1 (C-6), 18.0 (C-30), 15.3 (C-25), 15.2 (C-26), 14.7 (C-24), 13.7 (C-27).

Oleanolic acid (12)—White powder; $\left(\mathrm{C}_{30} \mathrm{H}_{48} \mathrm{O}_{3}\right):{ }^{1} \mathrm{H}$ NMR $\left(300 \mathrm{MHz}, \mathrm{CDCl}_{3}\right)$ $\delta_{\mathrm{H}}: 5.31$ ( $\left.m, \mathrm{H}-12\right), 3.23$ ( $\left.m, \mathrm{H}-3\right), 2.84$ ( $\left.m, \mathrm{H}-19\right), 2.80$ ( $\left.m, \mathrm{H}-18\right), 0.98\left(s, 23-\mathrm{CH}_{3}\right)$, $0.75\left(s, 24-\mathrm{CH}_{3}\right), 0.92\left(s, 25-\mathrm{CH}_{3}\right), 0.77\left(s, 26-\mathrm{CH}_{3}\right), 1.13\left(s, 27-\mathrm{CH}_{3}\right), 0.90(s$, 29- $\left.\mathrm{CH}_{3}\right), 0.92\left(s, 30-\mathrm{CH}_{3}\right) ;{ }^{13} \mathrm{C} \mathrm{NMR}\left(75 \mathrm{MHz}, \mathrm{CDCl}_{3}\right) \delta_{\mathrm{C}}: 182.9(\mathrm{C}-28), 143.7$ (C-13), 122.8 (C-12), 79.0 (C-3), 55.3 (C-5), 47.8 (C-9), 46.6 (C-17), 46.0 (C-19), 41.1 (C-14), 41.7 (C-18), 39.4 (C-8), 38.9 (C-4), 38.5 (C-1), 37.2 (C-10), 33.9 (C-21), 33.2 (C-29), 32.7 (C-7), 32.7 (C-22), 32.7 (C-20), 27.3 (C-23), 27.8 (C-15), 28.2 (C-2), 26.1 (C-27), 23.5 (C-30), 23.1 (C-16), 23.7 (C-11), 18.4 (C-6), 17.3 (C-26), 15.6 (C-24), 15.4 (C-25).

Ergosta-6,22-diene-3,5,8-triol (13)-White powder; $\left(\mathrm{C}_{28} \mathrm{H}_{46} \mathrm{O}_{3}\right):{ }^{13} \mathrm{C}-\mathrm{NMR}$ $\left(150 \mathrm{MHz}, \mathrm{CDCl}_{3}\right): \delta_{\mathrm{C}} 135.5(\mathrm{C}-6), 135.3(\mathrm{C}-22), 132.4(\mathrm{C}-23), 130.9(\mathrm{C}-7), 82.3$ (C-5), 79.6 (C-8), 66.6 (C-3), 56.3 (C-17), 51.8 (C-14), 51.2 (C-9), 44.7 (C-13), 42.9 (C-24), 39.9 (C-20), 39.4 (C-11), 37.1 (C-10), 37.0 (C-4), 34.8 (C-1), 33.2 (C-25), 30.2 (C-2), 28.8 (C-15) 23.5 (C-12), 21.0 (C-21), 20.8 (C-16), 20.1 (C-26), 
19.8 (C-28), 18.3 (C-19), 17.7 (C-27), 13.0 (C-18).

Stigmastane-3,6-dione (14)-White powder; $\left(\mathrm{C}_{29} \mathrm{H}_{48} \mathrm{O}_{2}\right):{ }^{1} \mathrm{H}-\mathrm{NMR}(500 \mathrm{MHz}$, Pyridine- $\left.d_{5}\right) \delta_{\mathrm{H}}: 2.64(m, \mathrm{H}-5), 2.29(m, \mathrm{H}-4 \mathrm{a}), 2.42(m, \mathrm{H}-4 \mathrm{~b}), 2.37$ ( $\left.m, \mathrm{H}-7 \mathrm{a}\right), 2.08$ ( $m, \mathrm{H}-1 \mathrm{~b}), 2.05$ ( $m, \mathrm{H}-2 \mathrm{a}), 2.05$ ( $m, \mathrm{H}-12 \mathrm{a}), 2.00$ ( $m, \mathrm{H}-7 \mathrm{~b}), 1.77$ ( $m, \mathrm{H}-16 \mathrm{a}), 1.73$ ( $m, \mathrm{H}-8), 1.64$ ( $m, \mathrm{H}-2 \mathrm{a}), 1.64$ ( $m, \mathrm{H}-12 \mathrm{~b}), 1.64$ ( $m, \mathrm{H}-25), 1.60$ ( $m, \mathrm{H}-11 \mathrm{a}), 1.43$ ( $m, \mathrm{H}-23 \mathrm{a}), 1.37$ ( $m, \mathrm{H}-9), 1.30$ ( $m, \mathrm{H}-11 \mathrm{~b}), 1.26$ ( $m, \mathrm{H}-20), 1.25$ ( $m, \mathrm{H}-1 \mathrm{a}), 1.25$ (m, H-17), 1.21 (m, H-22a), 1.18 (m, H-16b), 1.16 (m, H-14), 1.14 (m, H-28), 1.08 ( $m, \mathrm{H}-15), 1.00$ ( $m, \mathrm{H}-23 \mathrm{~b}), 0.92(m, \mathrm{H}-24), 0.91\left(d ; 6.4 \mathrm{~Hz}, 29-\mathrm{CH}_{3}\right), 0.90$ ( $m, \mathrm{H}-22 \mathrm{~b}), 0.87$ (br s, 21- $\left.\mathrm{CH}_{3}\right), 0.83\left(s, 18-\mathrm{CH}_{3}\right), 0.82\left(d ; 3.2 \mathrm{~Hz}, 26-\mathrm{CH}_{3}\right), 0.80$ (br s, 27- $\left.\mathrm{CH}_{3}\right), 0.66\left(s, 19-\mathrm{CH}_{3}\right) ;{ }^{13} \mathrm{C}-\mathrm{NMR}\left(125 \mathrm{MHz}\right.$, Pyridine- $\left.d_{5}\right) \delta_{\mathrm{C}}: 211.8(\mathrm{C}-3)$, 209.4 (C-6), 56.6 (C-5), 55.8 (C-17), 55.3 (C-14), 52.5 (C-9), 45.8 (C-7), 45.1 (C-24), 42.3 (C-13), 40.6 (C-10), 38.6 (C-1), 37.4 (C-8), 37.2 (C-12), 36.7 (C-4), 36.2 (C-2), 35.3 (C-20), 33.1 (C-22), 28.4 (C-25), 27.7 (C-16), 25.3 (C-15), 23.3 (C-23), 22.3 (C-28), 20.9 (C-11), 19.0 (C-26), 18.2 (C-27), 17.9 (C-21), 11.7 (C-29), $11.3(\mathrm{C}-19), 11.2(\mathrm{C}-18)$.

$\beta$-sitosterol (15) + stigmasterol (16): White crystal from methanol: $\mathrm{mp}$ : $135^{\circ} \mathrm{C}-137^{\circ} \mathrm{C}$ [lit. $134^{\circ} \mathrm{C}-136^{\circ} \mathrm{C}$ ] [39].

Stigmasterol 3-O- $\beta$-D-glucoside (17) - White powder; $\left(\mathrm{C}_{35} \mathrm{H}_{58} \mathrm{O}_{6}\right):{ }^{1} \mathrm{H}-\mathrm{NMR}$ $\left(600 \mathrm{MHz}\right.$, Pyridine- $\left.d_{5}\right) \delta_{\mathrm{H}}: 5.22(m, \mathrm{H}-22), 5.09(m, \mathrm{H}-23), 5.05\left(d, 7.7 \mathrm{~Hz}, \mathrm{H}-1^{\prime}\right)$, 4.58 ( $m, \mathrm{H}-6 \mathrm{a}), 4.40$ ( $m, \mathrm{H}-6 \mathrm{~b}), 4.33$ ( $m, \mathrm{H}-3), 4.26$ ( $m, \mathrm{H}-4), 4.05$ (m, H-2), 3.99 (m, H-3), 3.96 ( $m, \mathrm{H}-5), 2.05$ (m, H-20), 2.04 (m, H-2), 2.03 (m, H-7a), 1.98 ( $m, \mathrm{H}-12), 1.82$ ( $m, \mathrm{H}-14), 1.81$ ( $m, \mathrm{H}-16), 1.74$ ( $m, \mathrm{H}-7 \mathrm{~b}), 1.70$ ( $m, \mathrm{H}-1), 1.60$ (m, H-15), 1.59 (m, H-24), 1.55 (m, H-8), 1.54 (m, H-25), 1.43 (m, H-28), 1.26 ( $m, \mathrm{H}-17), 1.24(m, \mathrm{H}-9), 1.22(m, \mathrm{H}-4), 1.21(m, \mathrm{H}-11), 1.09\left(d, 6.5 \mathrm{~Hz}, 21-\mathrm{CH}_{3}\right)$, $0.92\left(d, 6.6 \mathrm{~Hz}, 27-\mathrm{CH}_{3}\right), 0.90(d, 7.3 \mathrm{~Hz}, \mathrm{H}-29), 0.87\left(d ; 7.1 \mathrm{~Hz}, 26-\mathrm{CH}_{3}\right), 0.74(s$, 19- $\left.\mathrm{CH}_{3}\right), 0.59\left(s, 18-\mathrm{CH}_{3}\right) ;{ }^{13} \mathrm{C}-\mathrm{NMR}\left(150 \mathrm{MHz}\right.$, Pyridine- $\left.d_{5}\right) \delta_{\mathrm{C}}: 139.9(\mathrm{C}-5)$, 139.1 (C-22), 130.0 (C-23), 118.2 (C-6), 102.6 (C-1 ), 79.1 (C-3), 78.9 (C-3), 77.5 (C-5), 75.8 (C-2), 72.2 (C-4), 63.3 (C-6), 56.7 (C-17), 55.9 (C-14), 51.2 (C-24), 49.9 (C-9), 43.8 (C-13), 41.5 (C-20), 40.5 (C-4), 39.9 (C-12), 37.9 (C-1), 35.1 (C-10), 34.9 (C-2), 32.6 (C-25), 30.4 (C-7), 30.1 (C-8), 29.3 (C-16), 25.5 (C-28), 23.7 (C-15), 22.1 (C-11), 22.0 (C-21), 21.7 (C-26), 19.6 (C-27), 13.4 (C-19), 12.9 (C-29), 12.6 (C-18).

\section{Chemophenetic Significance}

In this study, seventeen secondary metabolites (Figure 1) were isolated from Rumex abyssinicus including one new ceramide (1), one phthalate derivative (2), six anthraquinones (3-8), four triterpenes (9-12) and five steroids (13-17). From all these isolated metabolites, it appears that compounds 2, 5, 8-17 are reported here for the first time from $R$. abyssinicus. All the anthraquinones described here have already been isolated from other species of the genus Rumex: Compounds 3, 4 and 6 were isolated from $R$. abysinica [40] $R$. japonicus [24], $R$. acetosa., $R$. acetosella L., $R$. confertus Willd., $R$. crispus L., $R$. hydrolapathum Huds. $R$. obtusifolius [41] and $R$. aquaticus [42], compounds 5 and 7 from $R$. japonicus [43], $R$. 
nepalensis [44], compound 8 from $R$. obtusifolius [45] and compounds $\mathbf{7}$ and $\mathbf{8}$ from $R$. acetosa [46]. Base to the fact that, anthraquinones represent one of the main classes of secondary metabolites isolated from the genus Rumex [14] [47], their presence in all of the above species allowed us to conclude that $R$. abyssinicus and the other species mentioned exhibit very close chemotaxonomic relationships. The isolation of a new ceramide (1) from this plant was not a surprising since the works carried out by Watanabe et al., (2011) reported glucosylceramides in $R$. obtusifolius. Although compounds 15 and 16 have already been isolated from the genus Rumex [26] [48] [49] [50] [51], stigmasterol 3-O- $\beta$ - $D$-glucoside (17), stigmastane-3,6-dione (14), ergosta-6,22-diene-3,5,8-triol (13) have never been isolated from the family Polygonaceae. Bis-(2-ethylhexyl) phthalate (DEHP) (2) isolated for the first time from Rumex genus has already been reported from Polygonaceae family especially from Polygonum runcinatum [52].

\section{Conclusion}

In conclusion, the chemical study of the whole plant of $R$. abyssinicus led to the isolation of one previously undescribed ceramide (1) and sixteen known (2-17) compounds which enriched the chemical diversities of the plant, genus Rumex and Polygonaceae family. Compounds 10-12 may demonstrate here the relationship between $F$. aubertii and other species belonging to other genera herbs of Polygonaceae. Unfortunately, the extracts showed no antibacterial activity against the strains tested while compounds were either not active or weakly active. It will be interesting in future studies to test these extracts as well as the compounds isolated on other microbial strains.

\section{Acknowledgements}

The authors are grateful to the University of Dschang for financing some consumables used in this work, to the "Institut de Chimie Moléculaire de Reims" for the spectroscopic analysis on NMR equipments of the PlAnet Platform. They are also grateful to the German Academic Exchange Service (DAAD) for the financial support granted through the project: Yaoundé-Bielefeld Graduate School of Natural Products with Antiparasite and Antibacterial Activities (YaBiNaPA, project no. 57316173) as well as and the Cameroonian Ministry of Higher Education.

\section{Conflicts of Interest}

The authors declare no conflicts of interest regarding the publication of this paper.

\section{References}

[1] Aslam, B., Wang, W., Arshad, M.I., Khurshid, M., Muzammil, S., Rasool, M.H., Nisar, M.A., Alvi, R.F., Aslam, M.A. and Qamar, M.U. (2018) Antibiotic Resistance: A Rundown of a Global Crisis. Infection and Drug Resistance, 11, 1645-1658. 


\section{https://doi.org/10.2147/IDR.S173867}

[2] Yadav, S. and Kapley, A. (2021) Antibiotic Resistance: Global Health Crisis and Metagenomics. Biotechnology Reports, 29, e00604.

https://doi.org/10.1016/j.btre.2021.e00604

[3] Anand, U., Jacobo-Herrera, N., Altemimi, A. and Lakhssassi, N. (2019) A Comprehensive Review on Medicinal Plants as Antimicrobial Therapeutics: Potential Avenues of Biocompatible Drug Discovery. Metabolites, 9, Article ID: 258.

https://doi.org/10.339/metabo9110258

[4] Raju, N.J. and Yesuf, E.A. (2010) Evaluation of Anthelmentic Activities of Rumex abyssinicus Jacq and Rumex nervosus Vahl. (Polygonaceae). International Journal of Pharmaceutical Sciences Review and Research, 5, 55-57.

[5] Mulisa, E., Asres, K. and Engidawork, E. (2015) Evaluation of Wound Healing and Antiinflammatory Activity of the Rhizomes of Rumex abyssinicus J. (Polygonaceae) in Mice. BMC Complementary and Alternative Medicine, 15, Article No. 341. https://doi.org/10.1186/s12906-015-0878-y

[6] Shifa, M., Abdissa, D. and Asere, T.G. (2021) Chemical Constituents of Rumex abyssinicus Roots and Evaluation of Its Antimicrobial Activities. Journal of the Turkish Chemical Society Section A, 8, 21-46.

[7] Ntemafack, A., Vijay, K.N., Mohd, A., Qazi, P.H., Sumit, G.G. and Sandip, B.B. (2020) Discovery of Helminthosporin, an Anthraquinone Isolated from Rumex $a b$ yssinicus Jacq as a Dual Cholinesterase Inhibitor. ACS Omega, 5, 1616-1624. https://dx.doi.org/10.1021/acsomega.9b03693

[8] Desta, K.T., Kim, G.S., Hong, G.E., Kim, Y.H., Lee, W.S., Lee, S.J., Jin, J.S., El-Aty, A.M.A., et al. (2015) Dietary-Flavonoid-Rich Flowers of Rumex nervosus Vahl: Liquid Chromatography with Electrospray Ionization Tandem Mass Spectrometry Profiling and In Vitro Anti-Inflammatory Effects. Journal of Separation Science, 38, 3345-3353. https://doi.org/10.1002/jssc.201500737

[9] Kerem, Z., Bilkis, I., Flaishman, M.A. and Sivan, L. (2006) Antioxidant Activity and Inhibition of $\alpha$-Glucosidase by trans-Resveratrol, Piceid, and a Novel trans-Stilbene from the Roots of Israeli Rumex bucephalophorus L. Journal of Agricultural and Food Chemistry, 54, 1243-1247. https://doi.org/10.1021/jf052436

[10] Kerem, Z., Regev-Shoshani, G., Flaishman, M.A. and Sivan, L. (2003) Resveratrol and Two Monomethylated Stilbenes from Israeli Rumex bucephalophorus and Their Antioxidant Potential. Journal of Natural Products, 66, 1270-1272. https://doi.org/10.1021/np030087c

[11] Bicker, J., Petereit, F. and Hensel, A. (2009) Proanthocyanidins and a Phloroglucinol Derivative from Rumex acetosa L. Fitoterapia, 80, 483-495.

https://doi.org/10.1016/j.fitote.2009.08.015

[12] Jang, D.S., Kim, J.M., Kim, J.H. and Kim, J. (2005) 24-nor-Ursane Type Triterpenoids from the Stems of Rumex japonicus. Chemical and Pharmaceutical Bulletin, 53, 1594-1596.

[13] Shahnaz, S., Mohammed, A. and Showkat, R.M. (2017) Chemical Constituents from the Aerial Parts of Rumex hastatus D. Don. Journal of Pharmaceutical and Biological Sciences, 5, 179-187.

[14] Vasas, A., Orbán-Gyapai, O. and Hohmann, J. (2015) The Genus Rumex: Review of Traditional Uses, Phytochemistry and Pharmacology. Journal of Ethnopharmacology, 175, 198-228. http://dx.doi.org/10.1016/j.jep.2015.09.001

[15] Tamokou, J.D., Chouna, J.R., Fischer-Fodor, E., Chereches, G., Barbos, O., Damian, G., Benedec, D., Duma, M., Efouet, A.P.N., Wabo, H.K., Kulate, J.R., Mot, A. and Silaghi-Dumitrescu, R. (2013) Anticancer and Antimicrobial Activities of Some An- 
tioxidant-Rich Cameroonian Medicinal Plants. PLoS ONE, 8, e55880. https://doi.org/10.1371/journal.pone.0055880

[16] Muganga, R., Angenot, L., Tits, M. and Frédérich, M. (2010) Antiplasmodial and Cytotoxic Activities of Rwandan Medicinal Plants Used in the Treatment of Malaria. Journal of Ethnopharmacology, 128, 52-57. http://dx.doi.org/10.1016/j.jep.2009.12.023

[17] Mabou, F.D., Ngnokam, D., Harakat, D. and Voutquenne-nazabadioko, L. (2015) New Oleanane-Type Saponins: Leptocarposide B-D, from Ludwigia leptocarpa (Onagraceae). Phytochemistry Letters, 14, 159-164. http://dx.doi.org/10.1016/j.phytol.2015.10.008

[18] Tagousop, C.N., Ngnokam, D., Harakat, D. and Voutquenne-Nazabadioko, L. (2018) Melantheraside A-E, Five Original Triterpenes with Natural Chloride or Oxime Group from the Aerial Parts of Melanthera elliptica O. Hoffm. Phytochemistry Letters, 26, 38-43 https://doi.org/10.1016/j.phytol.2018.05.007

[19] Dongmo, K.A., Nono, N.R., Kaaniche, F., Mawabo, K.I., Frese, M., Chouna, R.J., Nkenfou, N.C., Lenta, N.B., Ngnokam, D., Nkeng-Efouet-Alango, P. and Sewald, N. (2020) A-Type Doubly Linked Proanthocyanidin Trimer and Other Metabolites from Canthium venosum Fruits, and Their Biological Activities. Phytochemistry Letters, 36, 134-138. https://doi.org/1016/j.phytol.2020.01.0200

[20] Kengne, I.C., Feugap, L.D.T., Njouendou, A.J., Ngnokam, C.D.J., Djamalladine, M.D., Ngnokam, D., Voutquenne-Nazabadioko, L. and Tamokou, J.D.D. (2021) Antibacterial, Antifungal and Antioxidant Activities of Whole Plant Chemical Constituents of Rumex abyssinicus. BMC Complementary Medicine and Therapies, 21, Article No. 164. https://doi.org/10.1186/s12906-021-03325-y

[21] Teles, Y.C.F., Chaves, O.S., de Fátima Agra, M., et al. (2015) Chemical Constituents from Sidastrum paniculatum and Evaluation of Their Leishmanicidal Activity. Revista Brasileira Farmacognosia, 25, 363-368.

https://doi.org/10.1016/j.bjp.2015.02.002

[22] Maheshwari, R., Varahalarao, V., Sai, P.P., Dhevendar, K., Prabhakar, S., Sunil, M., Ramars, A. and Suryanarayana, M.U. (2017) Isolation and Characterization of Phthalates from Brevibacterium mcbrellneri that Cause Cytotoxicity and Cell Cycle Arrest. Excli Journal, 16, 375-387.

[23] Bulus, A., Ben, A.C., Tarfa, F.D., Oluwakanyinsola, A.S. and Ogbaji, J.I. (2015) Isolation and Analgesic Property of Lupeol from Diospyros mespiliformis Stem Bark. Journal of Medicinal Plants Research, 9, 813-819. https://doi.org/10.5897/JMPR2015.58777

[24] Guo, S., Feng, B., Zhu, R., Ma, J. and Wang W. (2011) Preparative Isolation of Three Anthraquinones from Rumex Japonicas by High-Speed Counter-Current Chromatography. Molecules, 16, 1201-1210. https://doi.org/10.3390/molecules16021201

[25] Basu, S., Ghosh, A. and Hazra, B. (2005) Evaluation of the Antibacterial Activity of Ventilago Madraspatana Gaertn., Rubia cordifolia Linn. and Lantana camara Linn.: Isolation of Emodin and Physcion as Active Antibacterial Agents. Phytotherapy Research, 19, 888-894. https://doi.org/10.1002/ptr.1752

[26] Hala, S.A.E., Abdel-Fattah, M.R. and Hussiney, A.H. (1995) Constituents of Plants growing in Qatar, Part XXVI. Phytochemical Investigation of Rumex vesicarius. International Journal of Chemistry, 6, 21-25. http://hdl.handle.net/10576/5252

[27] Mumtaz, A., Sultan, M., Muhammad, R.S., Ajmal, K., Umer, R., Umar, F., Farhat, U., Abdul, S., Muhammad, A., Majid, A., Manzoor, A. and Abdul, L. (2017) Neurologically Potent Molecules from Crataegus oxyacantha; Isolation, Anticholinesterase Inhibition, and Molecular Docking. Frontiers in Pharmacology, 8, Article ID: 327. 
https://doi.org/10.3389/fphar.2017.00327

[28] Kun, W., Wei, L., Kazuo, K., Yuping, P. and Yingjie, C.T.N. (2004) Complete ${ }^{1} \mathrm{H}$ and ${ }^{13} \mathrm{C}$ NMR Assignments of Two Phytosterols from Roots of Piper nigrum. Spectral Assignments and Reference Data. Magnetic Resonance in Chemistry, 42, 355-359. https://doi.org/10.1002/mrc.1347

[29] Ayeleso, T.B., Matumba, M.G. and Mukwevho, E. (2017) Oleanolic Acid and Its Derivatives: Biological Activities and Therapeutic Potential in Chronic Diseases. Molecules, 22, Article ID: 1915. https://doi.org/10.3390/molecules22111915

[30] Rivera, A., Benavides, O.L. and Rios-Motta, J. (2009) (22E)-Ergosta-6,22-diene$3 \beta, 5 \alpha, 8 \alpha$-triol: A New Polyhydroxysterol Isolated from Lentinus edodes (Shiitake). Natural Product Research, 23, 293-300. https://doi.org/10.1080/14786410802038671

[31] Zhang, C., Wang, X., Zhang, X., Zhang, Y., Xiao, H. and Liang, X. (2009) Bioassay-Guided Separation of Citreorosein and Other Oestrogenic Compounds from Polygonum cuspidatum. Phytotherapy Research, 23, 740-741. https://doi.org/10.1002/ptr.2619

[32] Kubo, I., Murai, Y., Soediro, I., Soetarno, S. and Sastrodihardjo, S. (1992) Cytotoxic Anthraquinones from Rheum pulmatum. Phytochemistry, 31, 1063-1065. https://doi.org/10.1016/0031-9422(92)80078-s

[33] Garg, H.S. and Agrawal, S. (1995) A Novel Sphingosine Derivative from the Sponge Spirastrella inconstans. Journal of Natural Products, 58, 442-445. https://doi.org/10.1021/np50117a016

[34] Bankeu, K.J.J., Dawé, A., Mbiantcha, M., Feuya, T.G.R., Ali, I., Tchuenmogne, T.M.A., Mehreen, L., Lenta, N.B., Ali, M.S. and Ngouela, S.A. (2017) Characterization of Bioactive Compounds from Ficus vallis-choudae Delile (Moraceae). Trends in Phytochemical Research, 1, 235-242

[35] Stothers, J.B. (1972) Carbon-13 NMR Spectroscopy. 1st Edition, Academic Press, New York.

[36] Jung, J.H., Lee, C.O., Kim, Y.C. and Kang, S.S. (1996) New Bioactive Cerebrosides from Arisaema amurense. Journal of Natural Products, 59, 319-322. https://doi.org/10.1021/np960201

[37] Wouamba, S.C.N, Happi, G.M., Lenta, B.N., Sewald, N. and Kouam, S.F. (2020) Vernoguinamide: A New Ceramide and Other Compounds from the Root of Vernonia guineensis Benth. and Their Chemophenetic Significance. Biochemical Systematic and Ecology, 88, Article ID: 103988. https://doi.org/10.1016/j.bse.2019.103988

[38] Kuete, V. (2010) Potential of Cameroonian Plants and Derived-Products against Microbial Infections: A Review. Planta Medica, 76, 1479-1491. https://doi.org/10.1055/s-0030-1250027

[39] Luhata, L.P. and Munkombwe, N.M. (2015) Isolation and Characterisation of Stigmasterol and B-Sitosterol from Odontonema Strictum (Acanthaceae). Journal of Innovations in Pharmaceuticals and Biological Sciences, 2, 88-96. https://doi.org/10.13140/RG.2.1.3689.7365

[40] Munavu, R.M., Mudamba, L.O. and Ogur, J.A. (1984) Isolation and Characterization of the Major Anthraquinone Pigments from Rumex abysinica. Planta Medica, 50, 111. https://doi.org/10.1055/S-2007-969640

[41] Wegiera, M., Smolarz, D.H., Wianowska, D. and Dawidowicz, L.A. (2007) Anthracene Derivatives in Some Species of Rumex L. Genus. Acta Societatis Botanicorum Poloniae, 76, 103-108. https://doi.org/10.5586/asbp.2007.013

[42] Orbán-Gyapai, O., Liktor-Busa, E., Kúsz, N., Stefkó, D., Urbán, E., Hohmann, J. and 
Vasas, A. (2017) Antibacterial Screening of Rumex Species Native to the Carpathian Basin and Bioactivity-Guided Isolation of Compounds from Rumex aquaticus. Fitoterapia, 118, 101-106. https://doi.org/10.1016/j.fitote.2017.03.009

[43] Kim, J.M., Jang, D.S., Lee, Y.M., Lee, G.Y. and Kim, J.S. (2008) Constituents of the fruits of Rumex japonicus with Inhibitory Activity on Aldose Reductase. Journal of Applied Biological Chemistry, 51, 13-16. https://doi.org/10.3839/jabc.2008.003

[44] Liang, H.X., Dai, H.Q., Fu, H.A., Dong, X.P., Adebayo, A.H., Zhang, L.X. and Cheng, Y.X. (2010) Bioactive Compounds from Rumex Plants. Phytochemistry Letters, 3, 181-184. https://doi.org/10.1016/j.phytol.2010.05.005

[45] Hossein, N., Neda, B., Mahbubeh, G., Jamshid, M. and Farahnaz, B. (2017) Anti-Candida Activities and GC-Mass Analysis of Seed Hydroalcohlic Extract of Rumex obtusifolius. Jundishapur Journal of Microbiology, 10, e13733.

https://doi.org/10.5812/jim.13733

[46] Ullah, H.M.A., Kim, J.H., Rehman, N.U., Kim, H.J., Ahn, M.J. and Chung, H.J. (2018) A Simple and Sensitive Liquid Chromatography with Tandem Mass Spectrometric Method for the Simultaneous Determination of Anthraquinone Glycosides and Their Aglycones in Rat Plasma: Application to a Pharmacokinetic Study of Rumex acetosa Extract. Pharmaceutics, 10, Article ID: 100. https://doi.org/10.3390/pharmaceutics10030100

[47] Kuruüzüm, A. and Demirezer, L.Ö. (1997) Rumex L. Türlerinin Kimyasal Bileşimi. Fabad Journal of Pharmaceutical Sciences, 22, 79-87.

[48] Dhekra, M., Dalel, B.F.T., Slim, T. and Mohamed, T. (2018) Combinational Effect of Rumex tingitanus (Polygonaceae) Hexane Extract and Bacillus thuringiensis $\delta$-Endotoxin against Spodoptera littoralis (Lepidoptera: Noctuidae). BioMed Research International, 2018, Article ID: 3895834. https://doi.org/10.1155/2018/3895834

[49] Orbán-Gyapai, O., Forgo, P., Hohmann, J. and Vasas, A. (2017) Phytochemical Investigation of Rumex thyrsiflorus Fingerh. Acta Biologica Hungarica, 68, 232-236.

[50] Nusrat, S., Muhammad, S., Sumaira, K., Madiha, S., Syed, M.H. and Farhat, J. (2017) Investigation of Genus Rumex for Their Biologicallyactive Constituents. Research Journal of Life Sciences, Bioinformatics, Pharmaceutical and Chemical Sciences, 6, 148-165. https://doi.org/10.26479/2017.0206.11

[51] Abdul, W.S., Mohammed, A., Kamran, J.N. and Shahnaz, S.H. (2015) New Aliphatic Ester, $\beta$-Sitosterol Diglucoside and Vesicaria Biflavones from the Seeds of Rumex vesicarius L. Acta Poloniae Pharmaceutica, 72, 965-971.

[52] Wang, L.N., Xu, B.X., Cao, P.X. and Liang, G.Y. (2009) Studies on the Chemical Constituents of Polygonum runcinatum Buch.-Ham. var. sinense Hemsl. Natural Product Research and Development, 21, 73-75. 